\title{
OSIRIS software: the Mask Designer Tool
}

\author{
J.I. González-Serrano \\ Instituto de Física de Cantabria (CSIC-Universidad de Cantabria) \\ Avda. de los Castros s/n, 39005 Santander, Spain \\ gserrano@ifca.unican.es \\ M. Sánchez-Portal \\ Universidad Pontifícia de Salamanca en Madrid, 28040 Madrid, Spain \\ H. Castañeda \\ Instituto de Astrofísica de Canarias, 38200 La Laguna, Tenerife, Spain \\ R. Quirk, E.D. de Miguel \\ GMV S.A., 28760 Tres Cantos, Madrid, Spain \\ and \\ M. Aguiar, J. Cepa \\ Instituto de Astrofísica de Canarias, 38200 La Laguna, Tenerife, Spain
}

Received __; accepted _ 


\begin{abstract}
OSIRIS is a Day One instrument that will be available at the $10 \mathrm{~m}$ GTC telescope which is being built at La Palma observatory in the Canary Islands. This optical instrument is designed to obtain wide-field narrow-band images using tunable filters and to do low-resolution spectroscopy in both long-slit and multislit modes. For the multislit spectroscopy mode, we have developed a software to assist the observers to design focal plane masks. In this paper we describe the characteristics of this Mask Designer tool. We discuss the main design concepts, the functionality and particular features of the software.
\end{abstract}

\title{
1. INTRODUCTION
}

OSIRIS (Optical System for Imaging and low/intermediate-Resolution Integrated Spectroscopy) is an instrument designed to obtain images and low-resolution spectra in the optical domain (from 3650 through 10,000 $\AA$ ). It will be installed at the Nasmyth focus of the 10m GTC telescope (Álvarez and Rodríguez-Espinosa 2004) at La Palma observatory, although it can be also mounted at the Cassegrain focus. A detailed account of the observing modes, optical design, and performance is given in Cepa et al. (2003).

OSIRIS is a versatile instrument offering many observing modes: (a) direct imaging with broad-band and narrow-band using tunable filters, (b) narrow-band with charge shuffling, (c) fast photometry, (d) long-slit spectroscopy, (e) multi-object spectroscopy, (f) nod-and-shuffle multi-object spectroscopy, and (g) fast spectroscopy. The main driver of the optical and mechanical design of OSIRIS has been the inclusion of tunable filters (TF) in order to obtain narrow-band imaging at an arbitrary wavelength with effective resolutions of around 5-60 A. In the spectroscopic modes, OSIRIS provides spectral resolutions $R=\lambda / \Delta \lambda$ 
of $250,500,1500$, and 2500 covering the full spectral range from 3650 to $10,000 \AA$ by using 11 grisms. Grisms can be rotated by $90^{\circ}$ to change the dispersion direction and allow for fast spectroscopic observations. The detector consists of a mosaic of two $2 \mathrm{k} \times 4 \mathrm{k}$ Marconi MAT-44-82 CCDs of $15 \mu \mathrm{m} /$ pixel providing a large field of view $(\sim 8.5 \times 8.5)$ and a pixel size of $0{ }^{\prime} 125$. There will be a gap of 8.3 arcsec parallel to the normal dispersion direction.

OSIRIS will be the first instrument using the technique of tunable filters in large telescopes. The potential of this observational mode has been described widely in the literature (e.g. Jones and Bland-Hawthorn (2001); Jones, Shopbell, and Bland-Hawthorn (2002); Bland-Hawthorn and Kedziora-Chudczer (2003); Glazebrook, et al. (2004)).

The next observational mode in complexity is the multi-object spectroscopy (MOS). This technique arrived at its maturity in the eighties, increasing significantly the number of objects that can be observed simultaneously. Together with advances in optical design, new coatings for the camera elements, large-format detectors, and new technologies in grisms and diffraction gratings, their use in large telescopes is opening new windows in astronomical research, especially in the developments of large scale surveys. Recently, the use of the technique of nod-and-shuffle allows to obtain spectra with optimal sky subtraction, making possible to observe deeper (Glazebrook and Bland-Hawthorn 2001).

Most of the observing modes need to block part of the FOV to allow either charge shuffling or slit and multislit spectroscopy. This blocking is done by using a mask located at the telescope focal plane. Masks are fabricated by cutting the necessary holes or slits in aluminium plates which are of $496 \times 480 \mathrm{~mm}$ in size, being the size of the imaging field of $422 \times 428 \mathrm{~mm}$. In standard MOS mode, the number of slits ( 8 arcsec long) that can fit in a single mask is around 60 and 100 at the highest and lowest spectral resolution, respectively.

In this paper we describe the software that has been developed to assist the observer to design OSIRIS masks. 


\section{OVERVIEW OF THE OSIRIS MASK SYSTEM}

The OSIRIS Mask System is the system in charge of storing and positioning masks and it is fully described in Militello et al. (2003). Figure 1 shows a general view of the Mask System. A cassette provides storage for 13 masks which are positioned at the focal plane by a Mask Loader Mechanism. The position accuracy of the masks over the telescope focal plane is of the order of $0.01 \mathrm{~mm}$.

Masks are slightly curved in cylindrical form in order to adapt to the geometry of the focal plane. Masks will be tooled in a flat plate and afterwards bent to fit the cylindrical shape over the mask frame.

\section{OVERVIEW OF THE MASK DESIGNER}

For each 10m-class telescope where an instrument with MOS capabilities is operating, a specific software for mask design has been developed. Examples are the FORS Instrument Mask Simulator (Hummel 2000), the VIMOS Mask Preparation Software (Bottini, Garilli, and Tresse 2001), and the Mask Design Program of FOCAS (Saito et al. 2003). Our software has been designed to provide similar capabilities to these programs, adapted to the special needs of OSIRIS.

The Mask Designer is a subsystem of the OSIRIS Observing Program Manager which is, in turn, integrated into the GTC Control System (Filgueira and Rodríguez 1998). The software has been designed using Unified Modelling Language (UML) and coded in Java programming language.

The OSIRIS Mask Designer is a plug-in application of the JSkyCat $^{1}$ package. In this

\footnotetext{
${ }^{1}$ http: //archive.eso.org/JSky
} 
way, the software uses classes from JSkyCat to manage FITS files, to perform coordinate transformations, and for graphical user interface.

The Mask Designer performs all the calculations needed to transform sky (RA and DEC) and CCD $(x, y)$ coordinates to coordinates over the mask. The final output of the Mask Designer is therefore a set of instructions for the machine in charge of cutting the holes in the mask plate. This means that accurate coordinate transformations are needed to convert from sky to telescope focal plane positions, and from CCD to focal plane positions. Also, atmospheric refraction and thermal expansion corrections should be taken into account. Figure 2 shows all the coordinate transformations needed to correct a sky position onto a CCD position which include: (1) atmospheric refraction, (2) gnomonic projection and geometrical distortion of the telescope focal plane, (3) transformation from telescope focal plane onto mask surface, (4) mapping from mask to CCD plane. Atmospheric refraction is computed following Filippenko (1982) and depends on wavelength, air pressure and temperature, as well as on zenith distance of the targets. Transformations between different surfaces are made using 5th-order polynomials whose coefficients will be obtained in the instrument commissioning phase. Taking into account the errors due to mask fabrication, mechanical flexures, thermal expansion, astrometric accuracy, and the fact that the mask shape does not match exactly the focal plane, we expect that the position of the slits over the targets (after acquiring the field using fiducial stars), will be better than a $6 \%$ of the slit width. For a nominal slit of 1.4 arcsec, this corresponds to a positional accuracy of 0.08 arcsec.

Two types of masks can be generated depending of the OSIRIS observing mode. In the standard MOS mode, slits can be located at any position over the physical area of the mask. The second observing mode is nod and shuffle which allows astronomers to subtract the sky emission by nodding the telescope between targets and sky while simultaneously 
CCD charge is shuffled up and down (e.g. Glazebrook and Bland-Hawthorn (2001)). In this mode slits can be smaller than in standard MOS mode.

The system performs the necessary checks for mask integrity, slit collision and spectra overlapping. In case of overlapping slits or spectra the software can assign slits to additional masks using a priority-based algorithm. The software also takes into account the gap in the detector plane and the edges of the mask plate which are disabled by the mask frame that holds the mask plate.

\section{DECOMPOSITION DIAGRAM}

Figure 3 is a diagram showing the high-level classes in which the Mask Designer system has been decomposed. These classes (UserInputHandler, FileHander, and MaskHandler) are in turn decomposed into individual classes. The class OSIRIS_Mask_Designer represents the whole system and contains all the functionality to fully produce masks. Composite class UserInputHandler holds the functionality for handling the user interaction with the system. FileHander class is in charge of all actions regarding files. The MaskHandler composite class contains the algorithms for processing the slit data, transforming coordinates, and producing valid mask configurations. Its decomposition diagram is shown in Fig. 3.

Algorithms for processing the slits and producing valid mask configurations belong to classes SlitProcessor and MaskProcessor. Classes SkyToMaskMapping and CCD_ToMaskMapping contain the functions needed to transform coordinates between the different systems involved.

Not all the operations are shown in this diagram as all classes are decomposed into many simpler functions.

The Mask Designer has been designed as a plug-in of JSkyCat application, which 
contains many other classes and packages required by the system. The most relevant JSky utilities and classes used by the OSIRIS Mask Designer are: WCSTransform to convert between image pixel and WCS (World Coordinate System) coordinates; FITSCodec for handling of FITS files; GUI classes for image display and related tasks.

\section{FEATURES OF THE MASK DESIGNER}

The OSIRIS Mask Designer is launched by starting the JSkyCat application and therefore, its functionality initially is identical to that of JSkyCat. All operations are invoked through the main JSkyCat menu bar (see Fig. 4).

\subsection{Configuration file}

For the different parameters related to a particular observation, such as date, hour angle, central coordinates of the field, grism, position of the grism (0 or 90 degrees for normal or fast modes, respectively), position angle of the mask onto the sky, expected observation temperature and pressure, expected fabrication temperature, maximum number of masks per pointing, the system implements a Configure Observation menu to read an XML file (Observation Configuration File). Other XML configuration files are needed by the system but they are not user configurable. These contain telescope and instrument parameters and information relevant to computations, such as the coefficients for coordinate transformations and grism properties. The main advantage of using XML files is that full independence from target platform is guaranteed. 


\subsection{Input options}

The software can process slits defined interactively by using an image displayed on the screen, via cursor. This image should either have absolute astrometry information on its header or it can be a raw frame taken with OSIRIS in broad-band mode. Alternatively, the user can provide a list of coordinates and slit properties (type, width, priority) that can be, in turn, either absolute equatorial coordinates or pixel coordinates from a raw broad-band image taken with OSIRIS. These options allow processing object lists obtained with, for instance, image detection software.

The mask designer can be run in four different modes:

- Defining slit positions interactively using a raw image taken with OSIRIS

- Defining slit positions interactively using an image containing absolute astrometric information in its header

- Using a list of RA and DEC coordinates

- Using a list of $x, y$ positions from a raw image taken with OSIRIS

\subsection{Slits}

Three different types of slits can be defined: (a) rectangular, (b) circular, and (c) curved slits. Circular slits are used mainly (but not exclusively) to define fiducial holes. A minimum of three fiducial slits must be defined, normally to include bright stars in the field, in order to acquire the field. The type of the slit is defined by launching the Slit Window (see Fig. 5) and can be changed at any moment during the process of defining the slits. Also, the slit dimensions can be changed by selecting a slit already defined. Slits can be deleted either one by one or all at once. 
A special feature of this software is the ability of defining curved slits (available only in interactive processing). These are arc-shaped slits and may be useful for taking spectra of special targets such as, for instance, galaxy arcs produced by gravitational lensing.

\subsection{Overlapping and integrity check}

Integrity checks are made to avoid placing slits too close to mask borders or to other slits which may cause the mask to break at the fabrication process. Once all the slits are successfully configured the system processes the information. Several masks can be generated up to a maximum value specified by the user. The system will try to place as many slits as possible in the first mask. Slits are placed in additional masks depending on their priority, which is set by the user. Overlapping slits or slits producing integrity conflicts are placed onto the second mask and so forth until the maximum number of masks is reached. A residual mask is produced containing conflicting or out-of-bounds slits. This mask will not be sent to fabrication, being its only purpose to inform the user.

Conflicting and out-of-bounds slits are highlighted on the display using a color code to help the user to re-design the mask.

\subsection{Output}

The output consists of several files containing all the information required to build the masks. The user can save the work after masks have been processed. Saved files can be retrieved for later processing if needed. There are three output files, two of them in FITS format and the third in a format that will be read by the cutting machine software. A FITS file called ODF (Object Definition File) includes all the slits defined by the user, including invalid ones. The file contains the observation and telescope data, and, for all the generated 
masks, slit type and slit positions. A separate storage is provided in the ODF file to keep the fiducial slit information. This file can be handled by the Mask Designer to allow the user to modify the design in new sessions.

A second FITS file, called MDF (Mask Definition File), is generated for each mask. There is one file per mask containing only the slit information corresponding to that mask. A MDF can be saved also for the Residual Mask, although in that case, fiducial slits information is lost. The third file is the so-called Mask Cutter File and contains instructions to be read by the cutting machine to fabricate physically the masks.

The user has the option of printing a graphic view of each mask, containing the defined slits, the fiducial holes and, if wanted, rectangles marking where the spectra will appear in the image. An example is shown in Figure 6.

\section{CONCLUSIONS}

We have designed and developed an application to design focal plane multislit masks

for the instrument OSIRIS. Its functionality has been tested and the user requirements have been achieved. The software takes into account all the effects that could affect an accurate slit positioning over the mask plates, and allows different input scenarios to design OSIRIS masks. It has been designed as a JSky plug-in offering an easy user interface.

Only during the instrument commissioning phase will be possible to test the software in real conditions. A critical issue is the fine tuning of the parameters involved in coordinate transformations. In particular, the coefficients to convert from telescope focal plane to detector and from sky to telescope focal plane will be obtained using pin-hole masks and observations of astrometric fields. Commisioning phase for OSIRIS at the GTC is planned to start in 2006 . 
Figure 1 has been kindly provided by Lorenzo Peraza. This work has been supported by the Spanish Plan Nacional de Astronomía y Astrofísica under grants AYA2000-2688-E, AYA2002-03326, and AYA2002-01379. 


\section{REFERENCES}

Álvarez, P. and Rodríguez-Espinosa, J.M. 2004, Proceedings of the SPIE, vol. 5489, 583

Bland-Hawthorn, J. and Kedziora-Chudczer, L. 2003, PASA, 20, 242

Bottini, D., Garilli, B, Tresse, L. 2001, in ASP Conf. Ser. 238, Astronomical Data Analysis Software and Systems X, ed. F.R. Harnden, Jr., F.A. Primini, \& H.E. Payne (San Francisco: ASP) 455

Cepa, J. et al. 2003, Proceedings of the SPIE, vol. 4841, 1739

Filgueira, J.M. and Rodríguez, D. 1998, Proceedings of the SPIE, vol. 3351, 2

Filippenko, A.V. 1982, PASP, 94, 715

Glazebrook, K. and Bland-Hawthorn, J. 2001, PASP, 113, 197

Glazebrook, K., Tobber, J., Thomson, S., Bland-Hawthorn, J., Abraham, R. 2004, AJ, 128, 2752

Hummel, W. 2000, Proceedings of the SPIE, vol 4010, 190

Jones, D.H. and Bland-Hawthorn, J. 2001, ApJ, 550, 593

Jones, D.H., Shopbell, P.L., Bland-Hawthorn, J. 2002, MNRAS, 329, 759

Militello, C., Correa, S., Pérez, J., Fuentes, F.J., Pérez de Taoro, R., Cepa, J., Peraza, L. 2004, Proceedings of the SPIE, vol. 4841, 1515

Saito, Y., et al. 2003, Proceedings of the SPIE, vol. 4841, 1180 
Fig. 1. - General view of the OSIRIS Mask System showing the focal plane bench, the mask frame, and the mask cassette with capacity for 13 masks

Fig. 2.- Schematic view of the coordinate transformations involved in the design of a slit mask

Fig. 3.- OSIRIS Mask Designer decomposition diagram showing, at the highest level, the classes in which the Mask Designer is decomposed. A further class decomposition is shown here for class MaskHandler, which is in charge of the most important functions of the application

Fig. 4.- Mask Designer Menu selection in JSkyCat

Fig. 5.- Slit Window used to define and change slit properties

Fig. 6.- Example of output showing the slits and the predicted spectra over the detector area of OSIRIS. There are 68 slits including four fiducial circular holes and a curved slit. The horizontal band at the middle of the plot represents the gap between the two CCD detectors 
Keywords: telescopes - instrumentation: spectrographs 
This figure "Fig1.jpg" is available in "jpg" format from: http://arXiv.org/ps/astro-ph/0602507v1 
This figure "Fig2.jpg" is available in "jpg" format from: http://arXiv.org/ps/astro-ph/0602507v1 


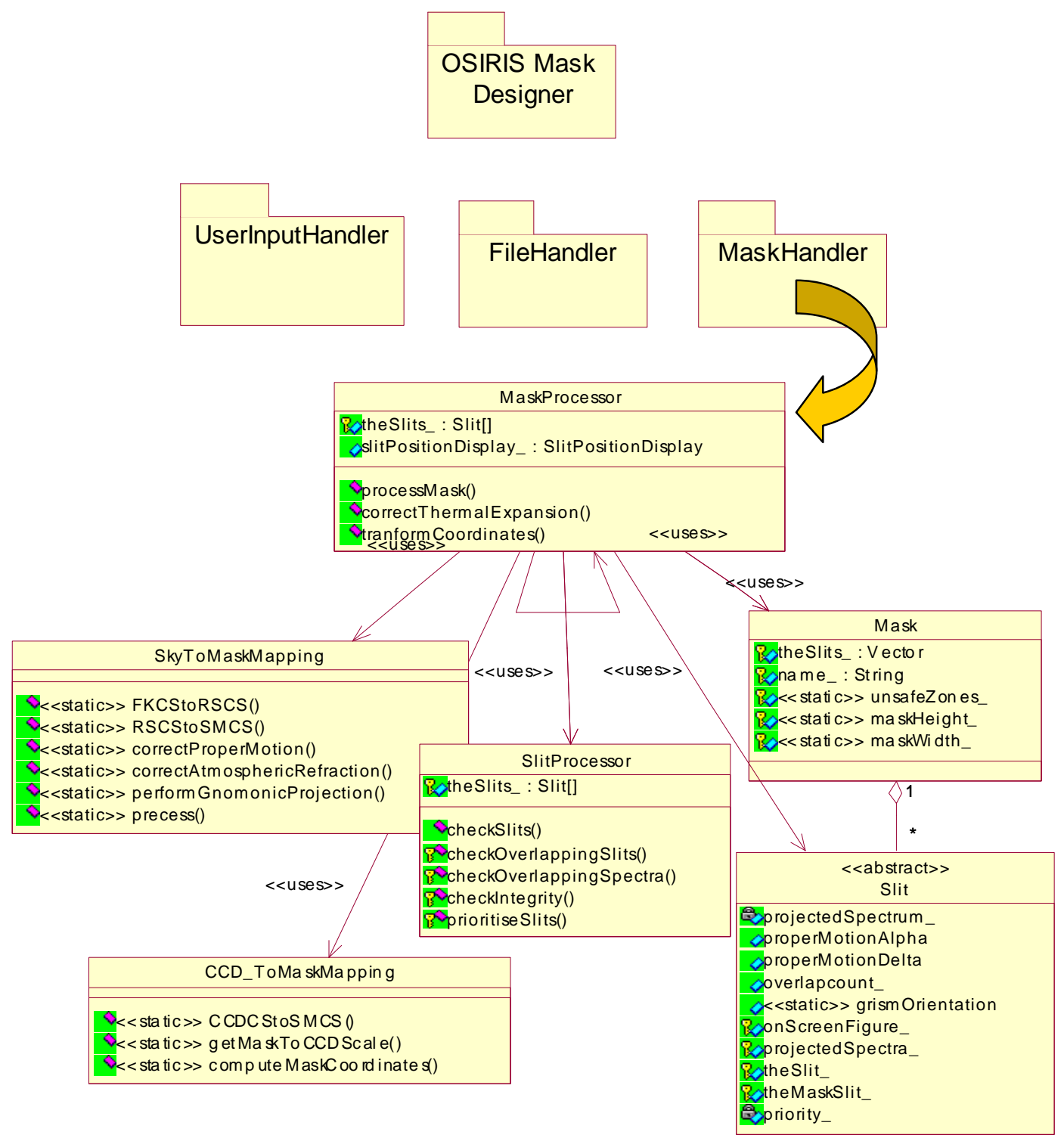


This figure "Fig4.jpg" is available in "jpg" format from: http://arXiv.org/ps/astro-ph/0602507v1 
This figure "Fig5.jpg" is available in "jpg" format from: http://arXiv.org/ps/astro-ph/0602507v1 
Mask Name: Mask1

Mask Platescale: 1.2133

Slit count: 68

Pos Angle (degrees): 90

Printed On: 30.04.2005 09:44:33 WEST

Image Centre: 00:17:30.5, +15:54:28 J2000



